\title{
ON-LINE DETERMINATION OF AGGREGATE SI E AND MORPHOLOGY IN SUSPENSIONS
}

\author{
Michel Cournil, Frédéric Gruy and Patrick Cugniet
}

\author{
Ecole des Mines de Saint-Etienne, Dept SPIN, URA CNRS 2021 \\ 158, Cours Fauriel, 42023 Saint-Etienne Cedex 2 (France)
}

\begin{abstract}
Information about the aggregation state of fine particles is an important element for process control, product quality monitoring and fundamental understanding in many cases of industrial slurries. hen aggregates are small or fragile objects, their ithdra al is difficult and off-line characterization may be a source of error. This ork deals ith the application of different in line methods to the characterization of silica aggregate size and morphology. These methods are based on turbidimetry. One of them consists of the analysis of the turbidity fluctuations and is operated on a commercial instrument. The other one uses the aggregate settling velocity hich is determined by turbidimetry too, ho ever ith a home-made apparatus. This ork gives us the opportunity to define morphological models for small aggregates and to calculate their drag coefficient. Thanks to these models, the aggregate morphological characteristics and the number of their constituting particles can be derived from the experimental results. Agreement bet een the different methods is examined and discussed.
\end{abstract}

\section{INTRODUCTION}

Contrary to agglomerates hich are commonly cemented by crystalline bridges hich confer them a rigid and solid structure, aggregates are most of the time small and fragile objects. In particular, in most experiments, their size does not exceed a maximum value hich results either from the dynamic balance bet een aggregation and fragmentation or from zero aggregation efficiency beyond a certain size [1-2]. For fundamental reasons as ell as for process monitoring purpose, real time kno ledge of the aggregate size and morphology may be very useful. For these determinations, both on-line and in-line methods can be envisaged. Off-line methods, ho ever, do not ensure isokinetic ithdra al, particularly, for small particles, and may damage fragile aggregates. In situ techniques ould be certainly ideal; ho ever, only very fe exist and they need to be validated. Turbidimetry has been proved to be a particularly efficient method for in situ particle size measurements [3], particularly in aggregating systems [1]. Turbidimetry is based on light scattering by suspensions and rests on the Mie theory [4].Ho ever, the scattering properties of small aggregates of micronic particles, for instance, is not completely kno $\mathrm{n}$ and requires additional research. Since the orks of essely et al. [5], analysis of turbidity fluctuations has been sho $\mathrm{n}$ as particularly interesting for the determination of particle number and size; this principle has given rise to a commercial instrument (Aello 4000).

The present ork is devoted to the characterization of aggregates of silica formed in a stirred vessel. Aggregation itself has been studied by in situ turbidimetry [6-7]. Final aggregates are characterized both using turbidity fluctuations and settling velocity measurements. Interpretation of these data requires preliminary theoretical tasks: morphological modelling of the aggregates and calculation of their light scattering section and drag coefficient. Comparison bet een the morphology and size respectively given by each method is presented and commented on.

\section{EXPERIMENTAL PART}

\section{Materials tec niques and procedure}

Aggregation experiments are performed on samples of monodisperse silica spheres $(1.5 \mu \mathrm{m}$ in diameter, Geltech Inc products). Aggregation is studied in ater at pH values 2 to 4 . 
The reactor used for this study of aggregation is a stirred tank the diameter of hich is $120 \mathrm{~mm}$ (Figure 1). This reactor is equipped ith four baffles. Liquid depth in the vessel is equal to diameter. Bottom part of the tank is rounded. Agitation is ensured by a four bladed 45 Teflon impeller of diameter $60 \mathrm{~mm}$. Temperature is kept constant at $25.00 \mathrm{C} \quad 0.01 \mathrm{C}$ by a double- all jacket. The reactor is fitted ith an optical system to measure in situ the suspension turbidity in the avelength range $350 \mathrm{~nm}-800 \mathrm{~nm}$. Details can be found in [6].

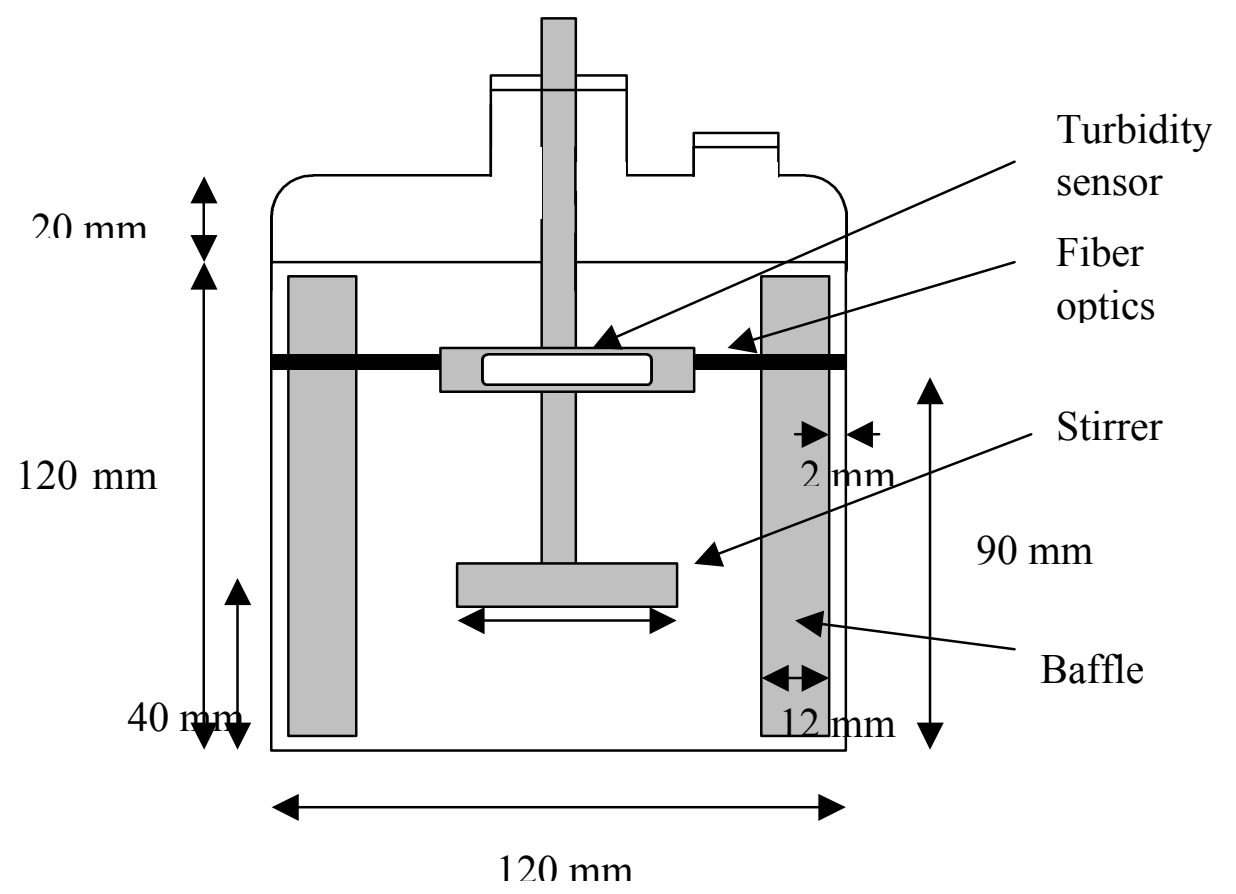

Figure 1. Schematic representation of the aggregation reactor

Turbidity $\tau$ expresses the extinction phenomenon of an incident light beam due to light scattering by solid particles; it is defined by relation:

$$
\tau \lambda_{0} \quad \frac{1}{L_{o p t}} \ln \frac{I_{0} \lambda_{0}}{I \lambda_{0}}
$$

in hich $\lambda_{0}$, is the avelength, $I_{0}\left(\lambda_{0}\right)$ the intensity of the incident beam and $I\left(\lambda_{0}\right)$ the intensity of the transmitted beam after an optical path of length $L_{\text {opt }}$.

Turbidity depends on the mean diameter $d_{p}$ particle density function $f$ according to the integral:

$$
\tau \lambda_{0} \quad C_{0} d_{\text {ext }} f d_{p} \mathrm{~d} d_{p}
$$

Particle extinction section $C_{e x t}$ is derived from the Mie theory [4]; its calculation is relatively easy for spherical, compact, or large particles, ho ever much more delicate for small, non compact aggregates [8]. From equation (2), it appears that variations in the particle density function $f$ result in turbidity variation. For instance, in the case of $1.5 \mathrm{~m}$ silica particles in ater, turbidity decreases ith aggregation, hereas it increases in the case of 0.5 $\mathrm{m}$ particles. hen particles leave the measurement cell, due to settling for instance, turbidity decreases obviously. These characteristics ill be exploited later on. 
Aello 4000 measurement cell is external to the reactor and located on a by-recirculation loop; thus measurements are in line and not in situ as previously. This apparatus delivers the turbidity signal of the suspension located in its measurement cell, ho ever ith a special interest in the signal fluctuations around its mean value. From these data, $\mathrm{t}$ o parameters are calculated, mean extinction section $C_{\text {ext }}$ and particle number in the cell $n$. Compared to our in situ determinations, this measurement procedure is liable to damage the aggregates, ho ever this effect is certainly reduced because shear stress in the loop is considerably lo er than in the reactor and pumping conditions are relatively smooth. This is confirmed by the identical turbidity levels observed in the $t$ o experimental systems.

Aggregation of a $0.246 \mathrm{~g}$ silica sample is performed in the previous reactor at given stirring rate. hatever the experimental conditions, turbidity reaches a constant value after a fe tens of minutes at the maximum; this plateau is generally interpreted as a steady state of the aggregate size distribution in the system and is characterized by a maximum aggregate size. After $\mathrm{t}$ o hours, this means during this steady state, agitation is stopped; particle settling is clearly observed and turbidity decrease is recorded. In similar experiments, suspension is pumped to the Aello 4000 cell and characterized during the aggregation process and at its end.

\section{Principle o interpretation o $t$ e sedimentation experiments}

The turbidity probe is vertically located at the $t \quad o$-thirds of the vessel radius half ay bet een $\mathrm{t} \mathrm{o}$ baffles, and mounted at $4.8 \mathrm{~cm}$ from the upper surface of the liquid (Figure 2)

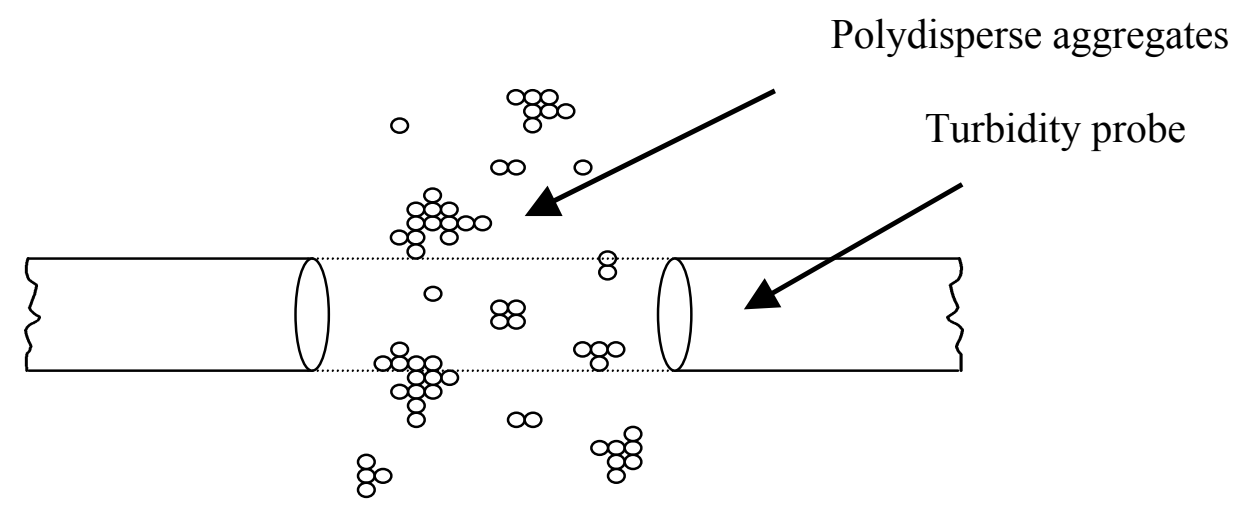

Figure 2. Aggregate settling as detected by the turbidity sensor
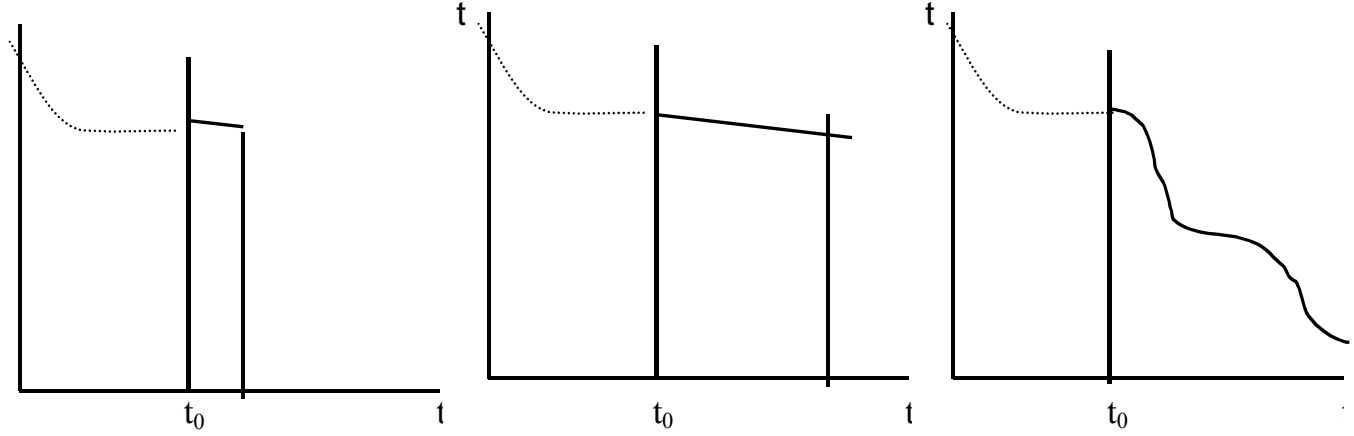

Figure 3.Turbidity variation during aggregate sedimentation,: a: large monodisperse aggregates; $b$ : small monodisperse aggregates; $c$ : polydisperse aggregates.

As soon as agitation is stopped, aggregates start settling and gradual decrease in turbidity is observed. In the ideal case of monodisperse aggregates, the turbidity signal keeps constant for a hile and sharply decreases only hen the aggregates initially located near the liquid 
surface have crossed the measurement indo . In the case of polydisperse aggregates, settling results in a classification of the aggregates according to their size and turbidity drop is not as sharp. Thus, according to the aggregate population nature, different turbidity plots against time are observed.

In Figure 3 e have represented the initial turbidity decrease due to aggregation in the stirred vessel in the time interval $\left[0, t_{0}=2\right.$ hours] then the turbidity decrease due to sedimentation. Cases a and $\mathrm{b}$ are relative to monodisperse aggregates; case $\mathrm{c}$, hich concerns polydisperse aggregates, is the most commonly observed.

Settling velocity of a silica spherical particle in ater is given by Stokes la :

$$
v_{1} \frac{2}{9} \rho \frac{a_{1} g}{\mu}
$$

in hich $a_{1}$ is the sphere radius, $g$, the gravity, $\mu$, the liquid dynamic viscosity and $\rho$ the density difference bet een silica and ater.

In the case of fractal-like aggregates, previous relation becomes:

$$
v \quad v_{1} \frac{i}{\beta}
$$

ith:

$$
\beta \frac{a_{i}}{a_{1}} \quad \frac{i}{S}{ }^{1 / D_{f}}
$$

in hich $i$ is the number of primary particles in the aggregate, $\Omega$ a corrective drag coefficient, $a_{i}$, the aggregate outer radius, $v$, its settling velocity. $D_{\mathrm{f}}$, the aggregate fractal dimension, is defined by this relation. $S$, structure factor, depends on $D_{\mathrm{f}}$ and is obtained from simulations. In turbulent aggregation, $D_{\mathrm{f}}$ is most of the time equal to 2.4 and $S=0.79$ [8].

From experimental curves similar to plots of Figure $3 \mathrm{a}$ and $3 \mathrm{~b}$, e can easily calculate $v$ by dividing the sensor mean depth from the top of the liquid (here $4.8 \mathrm{~cm}$ ) by the settling duration. Then $a_{1}$ is deduced from Eqn 3. This has been done for silica particles in conditions of non aggregation $(\mathrm{pH}=8)$ and good agreement has been found. For experimental plots similar to Figure $3 \mathrm{c}$, e have to choose a characteristic settling time to be able to determine the aggregate size. This $i l l$ be discussed further.

\section{Experimental results}

\subsection{Determination of the corrective drag coefficient}

As no model as available to determine drag coefficient $\Omega$ particularly for small aggregates,

e performed specific experiments. In order to use handy objects, e did experiments on $1 \mathrm{~mm}$ glass beads aggregates that e prepared ourselves [6]. Different sizes (2 to 80 particles) and morphologies ere obtained so. To keep Stokes settling conditions, e studied sedimentation in glycerol. Sedimentation time, hich is relatively long, as determined by direct observation of the settling objects, thus settling velocity as easily obtained. To apply Eqns 4 and 5, e needed to kno $a_{\mathrm{i}}$. e took it equal to the radius of the sphere hich had the same projected area as the aggregate. As the aggregates ere not necessarily isotropic, e obtained their projected surface area either from standard image analysis procedures of their photograph, or by direct calculation on the geometrical objects, kno ing the coordinates of the primary particles. Then $\Omega$ could be derived from Eqn 4 . In all cases, $\Omega$ as found practically equal to 1 ( $i$ th the specified choice of $a_{\mathrm{i}}$ ). 


\subsection{Settling of $1.5 \mathrm{~m}$ silica particles}

Using procedure described in section 2, e obtain the turbidity variation sho $\mathrm{n}$ in Figure 4 . Agitation as interrupted in the reactor after $t$ o hours aggregation. Plots of Figure 4 have the same shape as plot of Figure 3c. As aforesaid in section 2, characteristic time of settling should be chosen. Taken into account the existence of a main sedimentation ave on the different plots of Figure 4 e chose characteristic times corresponding to the end of this ave.

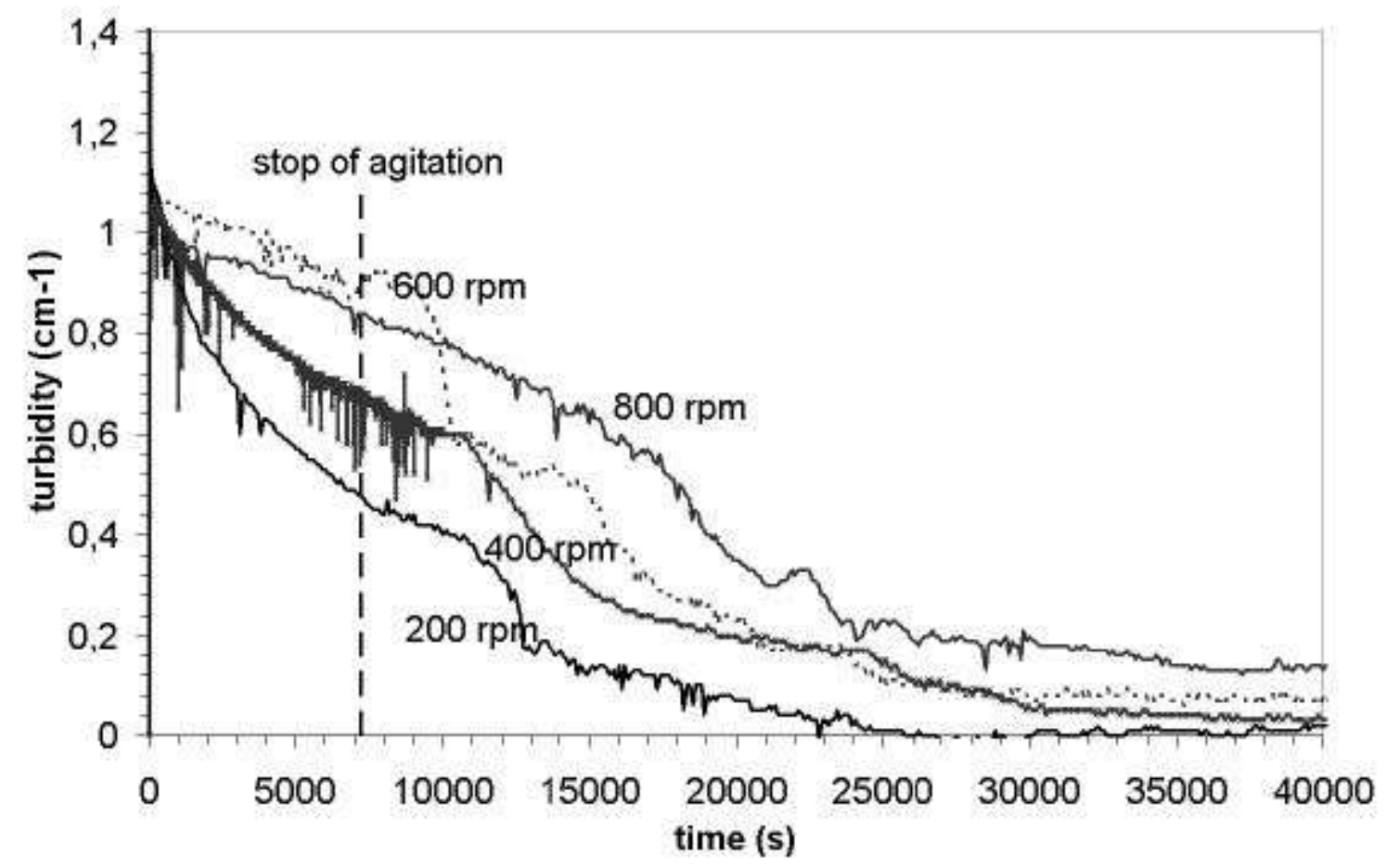

Figure 4: Settling of $1.5 \mu \mathrm{m}$ silica in ater $(\mathrm{pH} \quad 3, \lambda \quad 550 \mathrm{~nm})$

Table 1 sho $\mathrm{s}$ the different settling times and normalized settling velocity $\mathrm{v} / \mathrm{v}_{1}$ obtained for different stirring rates.

Table 1: Normali ed experimental settling velocity of silica aggregates formed at different stirring rates

\begin{tabular}{|ccc|}
\hline Stirring rate (rpm) & Settling time(s) & $v / v_{1}$ \\
\cline { 1 - 3 } 200 & 6000 & 5.8 \\
\hline 400 & 8000 & 4.4 \\
\hline 600 & 13000 & 2.7 \\
\hline 800 & 15400 & 2.3 \\
\hline
\end{tabular}

\subsection{Aello 4000 determinations}

As aforesaid, the Aello 4000 equipment allo ed us to obtain $C_{\text {ext }}$ and number $n$ of particles in the cell at any time, in particular, total number of silica particles $n_{0}$ is kno $\mathrm{n}$. This is the initial number of particles hich is measured at time zero prior to aggregation. Dividing $n_{0}$ by $n$ 
gives us $L$ the mean number of silica particles per aggregate. Data relative to the aggregationfragmentation steady state are reported in Table 2 for different stirring rates.

3.4. Image analysis

Attempts of aggregate removal after 2 hours aggregation have been made. After their ithdra al ith a pipette, the samples have been carefully dried, then observed ith a microscope coupled ith an image analyser ( eiss Axioskop microscope, magnification : $\mathrm{x}$ 500; video camera J C KY-F58; Leica - in soft are). Photographs of the removed samples have been processed in order to determine the number of primary particles per aggregate. Results are reported in Table 2.

Table 2: Estimation of the mean number of silica particles per aggregate

\begin{tabular}{|ccccc|}
\hline $\begin{array}{c}\text { Stirring rate } \\
(\mathrm{rpm})\end{array}$ & 200 & 400 & 600 & 800 \\
\hline $\left.\mathrm{C}_{\mathrm{ext}} \quad \mathrm{m}^{2}\right)$ & 23 & 14 & 6 & 5 \\
\hline$n$ & 210 & 400 & 1000 & 1500 \\
\hline$n_{0} / n(\quad L)$ & 28 & 15 & 6 & 4 \\
\hline $\begin{array}{c}\text { Particle } \\
\text { number } \\
(\text { from image } \\
\text { analysis) }\end{array}$ & 46 & $\ldots$ & 25 & 20 \\
\hline $\begin{array}{c}\text { Particle } \\
\text { number (from } \\
\text { settling data) }\end{array}$ & 20 & 15 & 5 & 5 \\
\hline
\end{tabular}

Table 3: Calculated values of normali ed $\left(v / v_{1}\right)$ settling velocity of aggregates for different fractal dimensions and particle numbers

\begin{tabular}{|c|ccccccccc|}
\cline { 2 - 8 } \multicolumn{1}{c|}{} & \multicolumn{6}{c|}{ Fractal dimension $D_{f}$} \\
\cline { 2 - 9 } & 2 & 2.2 & 2.3 & 2.4 & 2.5 & 2.6 & 2.7 & 2.9 \\
\hline 8 & 3.1 & 3.2 & 3.2 & 3.3 & 3.4 & 3.6 & 3.7 & 3.9 \\
\hline 16 & 4.3 & 4.6 & 4.8 & 5.0 & 5.2 & 5.4 & 5.7 & 6.2 \\
\hline 24 & 5.3 & 5.7 & 6.0 & 6.3 & 6.6 & 7.0 & 7.3 & 8.0 \\
\hline 32 & 6.0 & 6.6 & 7.0 & 7.5 & 7.9 & 8.3 & 8.8 & 9.7 \\
\hline
\end{tabular}

\section{DISCUSSION}

\section{Settling velocity calculations}

Using Equations (3) to (5) of section 2 and experimental determinations of the corrective drag coefficient (section 3.1), e can predict the normalized settling velocity of aggregates of given number of primary particles and given fractal dimension. These calculations are reported in Table 3 
No this table can be used as follo s:

i) the respective experimental normalized settling velocities of the aggregates formed at different stirring rates are extracted from Table 1;

ii) the respective closest values of normalized settling velocity are searched for in the columns $D_{\mathrm{f}}=2.4-2.5$ hich is the likely fractal dimension of the aggregates. In fact, the fractal dimension could have been obtained by image analysis of experimental samples, ho ever, only in the case of very large aggregates of small particles. This is not the case here. Thus, e selected the present values because they ere commonly found from previous experiments and simulations.

iii) the corresponding particle number in the aggregate is found after interpolation on data of Table 3 and then reported in last ro of Table 2.

e can no compare the estimations of particle number in small silica aggregates hich ere derived using the different methods.

i) Aello 4000 measurements and determinations from settling velocities are in good agreement and indicate that this particle number is very lo. Aello equipment is supposed to give reliable results for particles ranging bet een 1 and 250 micrometers in equivalent diameter;

ii) direct measurements on ithdra $\mathrm{n}$ samples are very different and certainly non representative. They confirm the ell kno $\mathrm{n}$ difficulty of isokinetic removal of small objects.

Similar studies ere performed on $0.5 \mathrm{~m}$ silica particles and lead us to the same conclusions [6]. The Aello 4000 apparatus still provided us ith correct results in spite of the lo value of the particle diameter.

\section{Maximum aggregate si e}

As e mentioned in the introduction of this paper, the existence of maximum aggregate size can be due to $t$ o main reasons : fragmentation or collision efficiency becoming zero beyond a critical size.

\subsection{Fragmentation}

The occurrence of breakage depends on the balance bet een the disaggregation effects due to the action of the fluid and the overall cohesion of the aggregate due to the interactions bet een primary particles. The hydrodynamic effects are of different nature according that the aggregate is larger or smaller than the Kolmogorov microscale. Only the latter case is compatible ith the experimental conditions of this study. It corresponds to a shear stress

originating from the local velocity gradient $\dot{\gamma}$ and acting on the aggregate. The breakage rate or the fragmentation kernel $K_{\mathrm{f}}$ is generally assumed to be proportional to $\gamma e^{\frac{\sigma}{\tau}}$, here $\sigma$ is the mean mechanical strength of the aggregate and $\tau_{s}$ is the mean shear stress. Thus, the breakage rate depends on the hydrodynamic conditions of the flo, via $\dot{\gamma}$ and on the characteristics of the aggregates: outer radius, fractal dimension, primary particle radius and cohesion force bet een $\mathrm{t}$ o primary particles (typically van der aals force).

In recent orks $[2,7-8]$ on aggregation of titanium dioxide or silica in similar conditions, ho ever, e came to the conclusion that such fragmentation kernels ere not able to interpret the maximum size observed in our experiments, thus e adopted the follo ing theoretical approach.

2.2. ero collision efficiency

This approach as especially developed by Brakalov [9].It is kno $\mathrm{n}$ that the collision efficiency bet een $t$ o equally sized spherical particles decreases ith the particle size. The decrease is sharper as the particles (aggregates) are porous. Other ise, the aggregate, hich 
results from $\mathrm{t}$ o smaller aggregates, can be too loose to survive. Brakalov sho $\mathrm{s}$ that it exists a maximum value for the aggregate size. From different experimental orks, it appears that the maximum particle size $a_{\mathrm{L}}$ depends on shear rate, according to relation:

$$
\frac{a_{L}}{a_{1}} \dot{\gamma}^{c^{\prime}}
$$

In recent orks on aggregation in the same reactor [2, 6, 7], e found values from 0.22 to 0.60 for exponent $c^{\prime}$. Moreover, after integrating the assumption of zero aggregation efficiency beyond this size in our models, e obtained good agreement bet een predicted and observed dynamics.

e recall that the gradient velocity as a function of the turbulent energy dissipation rate $\varepsilon_{\mathrm{m}}$ and kinematic viscosity $\mathrm{v}$ :

$$
\dot{\gamma} \frac{\varepsilon_{\mathrm{m}}}{\mathrm{v}}{ }^{\frac{1}{2}}
$$

Mean value of $\varepsilon_{\mathrm{m}}$ is given by the ell-kno $\mathrm{n}$ equation:

$$
\bar{\varepsilon}_{\mathrm{m}} \frac{N_{p} \omega^{3} D_{\mathrm{s}}^{5}}{V}
$$

in hich, $N_{\mathrm{p}}$ is the po er number, $D_{\mathrm{s}}$ the stirrer diameter, $\omega$ the rotation rate of the stirrer and $V$ the volume of the suspension..

Using the results of the present study (respectively Aello measurements and settling data from Table 2) and equations (5-8), e can no verify relation (6) in our case and determine exponent $c^{\prime}$. Good agreement is found, hich validates the mathematical form of Equation (6) and respective values of $c^{\prime}$ are: 0.24 and 0.19 . These values are of the same order of magnitude as previous results obtained on titanium dioxide aggregation [2].

\section{CONCLUSION}

This study clearly proves the interest of in situ or in line particle size determinations, especially based on turbidimetry, for aggregate or agglomerate characterization. Thanks to this procedure, small and fragile objects can be characterized in situ ithout taking the risk of damaging or non isokinetic ithdra als. Another interesting aspect of the Aello 4000 measurements is the possibility of quasi-instantaneous characterizations, allo ing in this to characterize rapidly changing media or objects. From a fundamental point of vie , this ork has allo ed us to model the settling velocity of small ramified objects, hich as not kno $n$ so far. The dependence on the aggregate particle number has been determined according to the stirring rate. Thanks to these data progress can be expected in the understanding of the aggregate fragmentation mechanisms.

\section{REFERENCES}

[1] Saint-Raymond, H., Gruy F., Cournil M., J. Colloid and Interf. Sci., 202 (1998), 238.

[2] Tontrup C., Gruy F., Cournil M., J. Coll. Inter. Sci., 229 (2000), 511-525.

[3] Cra ley G.M., Cournil M., Di Benedetto D., Po der Technol. 91 (1997), 197.

[4] an de Hulst H.C., Light Scattering by Small Particles (1957) iley, Ne York.

[5] essely, B., Altmann J., and Ripperger S., Chem. Eng. Science 19 (1996), 438.

[6] Cugniet P., PhD Dissertation, (2003), Etude de l'agrégation de particules solides en milieu non mouillant. Interprétation et modélisation, Ecole des Mines de Saint-Etienne (France).

[7] Cournil M., Gruy F., Cugniet, P., Congrès Français de Génie des Procédés (2003), Saint-Nazaire, submitted.

[8] Gruy, F., J. Colloid and Interf. Sci., 237 (2001), 28.

[9] Brakalov, L.B., Chem. Eng. Sci., 42 (1987), 2373. 